\title{
Application of a weighted average model to a psychophysical averaging task'
}

NORMAN H. ANDERSON

UNIVERSITY OF CALIFORNIA, SAN DIEGO

Ss lifted a sequence of six weights with instructions to judge their average heaviness. A model that took the judgment to be an average of the stimulus values, weighted for serial position, gave a satisfactory quantitative account of the data. The later weights in each sequence had greater influence on the judgment, a recency effect.

This article reports an experimental test of a simple model for serial integration of lifted weights. In the experimental task, $S$ lifts several weights in given order, then judges their average heaviness. This requires that he integrate the perceived heavinesses of the individual weights. A main theoretical issue concerns the way in which the integration is performed. The present hypothesis is that the judgment is simply an average of the perceived values of the stimuli, suitably weighted for serial position.

The present experiment is based on two previous reports (Anderson, 1964a, b) that describe the model and give an application to sequential number averaging. Several applications to impression formation have also been made (e.g., Anderson, 1962, 1965) although that task differs in important respects from the present weight averaging task.

The judgment $J$ of a sequence of lifted weights is assumed to be simply

$$
J=\Sigma w_{k} s_{k},
$$

where $s_{k}$ is the felt heaviness and $w_{k}$ the relative importance of the $\mathrm{kth}$ weight of the sequence. It should be noted that $s_{k}$ represents the scale value of the stimulus weight, $w_{k}$ its mathematical weight in the average.

Stimuli

The eight main experimental sets are shown in the left column of Table 1. The symbols $L$ and $H$ represent two successive weights of 2 and $6 \mathrm{oz}$, respectively, and are listed in the order of presentation. Since each weight always occurs twice in succession, these eight sets may be considered as a $2^{3}$ design, and this is convenient in the analysis.

Six additional experimental sets are listed in the right column of Table 1 . The first three consist of a pair of $4.5 \mathrm{oz}$ weights, denoted by $\mathrm{M}+$, combined in the three possible ways with two pairs of $\mathrm{L}$ weights. The last three consist of a pair of $3.5 \mathrm{oz}$ weights, denoted by $\mathrm{M}-$, combined similarly with two pairs of $\mathrm{H}$ weights.

Procedure

$S$ sat facing a vertical panel that concealed the $\mathrm{E}$ and the weights. The weights were lifted by making
Table 1

\begin{tabular}{lccc}
\multicolumn{4}{c}{ Table 1 } \\
Mean Heaviness Judgments of Six-weight Sequences \\
Sequence* & Response & Sequence* & Response \\
HHH & 19.60 & M+L L & 7.30 \\
HHL & 12.40 & L M+L & 8.40 \\
HLH & 13.75 & L LM+ & 9.45 \\
HLL & 6.95 & & \\
LHH & 14.10 & M-HH & 15.40 \\
LHL & 7.65 & HM-H & 14.05 \\
LLH & 8.45 & H HM- & 13.60 \\
LLL & 2.45 & &
\end{tabular}

* Each symbol represents two successive weights.

a short downward pull on a handle that led by a string and pulley to the rear of the panel. The rear end of the string held a small magnet and small magnets were also mounted on each weight. The placement of each weight produced a click that served as the signal to pull.

After instructions and five practice sets, the 14 experimental sets were given in mixed order. Responses were made orally on a scale from 1 (Very Light) to 20 (Very Heavy). The six weights of a set were presented at a uniform rate that took very nearly 30 sec. Twenty females from introductory psychology served as Ss.

Results

The mean heaviness judgment for each sequence of weights is shown in Table 1. These data exhibit a uniform recency effect, most readily seen in the right column. There the upper three sets have the same weights arranged in different orders. The closer that the heavier $M+$ pair is to the end of the sequence, the heavier is the judgment. The lower three sets show a similar recency effect. The $\mathrm{M}+$ and $\mathrm{M}-$ stimuli, it may be noted, serve as probe stimuli in a generalized order effect paradigm (Anderson, 1965).

The sets in the left column can be inspected for recency in the same way. For these sets, however, the design yields a serial position curve that provides a more compact view of the recency effect.

This serial position curve is shown by the three entries in the second column of Table 2. Each entry

Table 2 .

Serial position curve and test of goodness-of-fit of averaging model

\begin{tabular}{lccccc}
\multicolumn{3}{c}{ Moin Effects } & \multicolumn{3}{c}{ Interactions } \\
Source & Effect & s.e.m. & Source & Effect & s.e.m. \\
\hline First & 5.01 & .26 & FS & 0.11 & .26 \\
Second & 5.54 & .24 & FT & 0.39 & .25 \\
Third & 6.61 & .27 & ST & 0.21 & .25 \\
& & & FST & -0.01 & .29
\end{tabular}


is the difference in effect between an $\mathrm{H}$ pair and an $L$ pair at one of the positions in the sequence of weights. For example, 6.61 is the difference in judgment between those four sets with an $H$ pair and those four sets with an $L$ pair at the last position of the sequence.

This serial position curve shows uniform recency. There is a moderate increase between First and second, and a more marked increase between second and Third serial positions. Nevertheless, the First serial position has a very sizable effect of 5.01. Thus, the influence of the first two weights persists through the presentation of the four following weights.

The theoretical hypothesis at issue is that the judgment of a sequence is a weighted average of the felt heavinesses of the stimulus weights. If this hypothesis is true, then the statistical interactions of the $2^{3}$ design are zero. The algebraic values of these interactions, expressed as specific comparisons (Anderson, 1964b), are shown in the right half of Table 2. Each may be compared to its listed standard error $(\mathrm{df}=19)$, and none is significant. The $t$ of $\mathbf{1 . 5 6}$ for the FT interaction reflects the decrease in the effect of the Third serial position as measured by the difference between successive pairs of responses going downward in Table 1. This discrepancy, if real, may stem from an end-response tendency. It has not been observed in subsequent work in which end-anchor sets have been used more extensively. On the whole, therefore, the averaging model appears to provide a satisfactory account of the data.

\section{Discussion}

The present data give preliminary support to the averaging model for one class of psychophysical stimuli. If further work substantiates the model, it may then be put to work to scale the stimuli. This ability of the model to serve as a scaling procedure has been noted previously (Anderson, 1962), though some minor changes in experimental design and analysis would be required to take into account the serial position effect. For scaling purposes, of course, it would be desirable to use more than two stimuli at each serial position.

However, there is one theoretical complication that has been raised in a related connection (Anderson, 1966). As the model is ordinarily applied, it assumes that the subjective value of a stimulus is the same in any set, and success of the model is taken to reflect favorably on this assumption. But it is also possible to suppose that the stimulus values vary from one context to another, and that the judgment is an average of these context-modified values. This model will also satisfy the present test of fit if the stimulus values at a given position change as a fixed linear function of the context-free values of the other stimuli of that set. The present data, therefore, though they do support an averaging formulation, do not necessarily imply that the stimulus values are unaffected by context.

Up to a certain point, this theoretical complication does not invalidate the scaling procedure. Under either assumption the obtained scale values would be equivalent up to a linear transformation. The same equivalence would hold over serial position, which would be of particular relevance for scaling.

However, the theoretical complication does bear directly on the interpretation of the observed recency effect. If the felt heaviness of a given weight is constant, then the recency effect does stem from greater weight being associated with the later stimuli. However, context effects might be present so that, for instance, $\mathrm{L}$ is felt lighter in HHL than in LLL. Such a contrast effect would be predicted by Helson's (1964) adaptation-level theory. Contrast would also produce recency, but because of changed scale values, not because of differential weighting. The present data do not distinguish between these two possibilities. The problem is being studied by asking for judgments of the single components of the stimulus sequence.

\section{References}

Anderson, N. H, Application of an additive model to impression formation. Science, 1962, 138, 817-818.

Anderson, N. H. Note on weighted sum and linear operator models. Psychon. Sci., 1964, 1, 189-190.

Anderson, N. H. Test of a model for number-averaging behavior. Psychon. Sci., 1964, 1, 191-192.

Anderson, N. H. Primacy effects in personality impression formation using a generalized order effect paradigm. J. Pcrs. soc. Psychol., 1965, 1, 1-9.

Anderson, N. H. Component ratings in impression formations. Psychon. Sci., 1966, 6, 279-280.

Helson, H. Adaptation-level thcory. New York: Happer \& Row, 1964 .

\section{Notes}

1. This work was supported by NSF grants GB-1470 and GB-3913. I wish to thank Ann Jacobson for her assistance. 